Bro-cam: Improving game experience with empathic feedback using posture tracking

Peer-reviewed author version

TAN, Chiew Seng Sean; SCHOENING, Johannes; SCHNEIDER BARNES, Jan; LUYTEN, Kris \& CONINX, Karin (2013) Bro-cam: Improving game experience with empathic feedback using posture tracking. In: Persuasive Technology 2013, p. 222-233.

Handle: http://hdl.handle.net/1942/14555 


\title{
Bro-cam: Improving game experience with empathic feedback using posture tracking
}

\author{
Chiew Seng Sean Tan, Johannes Schöning, Jan Schneider Barnes, \\ Kris Luyten, Karin Coninx \\ Hasselt University \\ tUL - iMinds \\ Expertise Centre for Digital Media \\ Diepenbeek, Belgium \\ firstname. lastnameduhasselt.be
}

\begin{abstract}
In todays videogames user feedback is often provided through raw statistics and scoreboards. We envision that incorporating empathic feedback matching the player's current mood will improve the overall gaming experience. In this paper we present Bro-cam, a novel system that provides empathic feedback to the player based on their body postures. Different body postures of the players are used as an indicator for their openness. From their level of openness, Bro-cam profiles the players into different personality types ranging from introvert to extrovert. Empathic feedback is then automatically generated and matched to their preferences for certain humoristic feedback statements. We use a depth camera to track the player's body postures and movements during the game and analyze these to provide customized feedback. We conducted a user study involving 32 players to investigate their subjective assessment on the empathic game feedback. Semi-structured interviews reveal that participants were positive about the empathic feedback and Bro-cam significantly improves their game experience.
\end{abstract}

Keywords: Personalized Feedback, Posture Recognition, Persuasive Computing, Game experience.

\section{Introduction}

The recent push towards using gestural and motion gaming platforms (such as Microsoft Kinect, Sony PlayStation Move, and Nintendo Wii) uses the player's physical actions for the interaction with the game. Still, game feedback is presented in raw numbers and is not adapted to the user's mood. We expect that adapting the feedback to the current mood of the players will enhance the overall game experience as users have shown the preference for an empathetic agent with non-repetitive dialogs [3]. For example, it would be more appropriate to support users with encouraging comments after a game when they are slouching their shoulders instead of making harsh cynical comments. This is also motivated by recent work of Aviezer et al. [1]. They

Persuasive Computing 2013, p. 1, 2013.

(C) Springer-Verlag Berlin Heidelberg 2013 
have shown that body postures are typically used more often than other modalities for recognizing emotions. In their Science paper Aviezer et al. analyzed body cues and facial expressions of different professional tennis players. Their study showed that body cues, which dominate over facial expressions, are useful to discriminate between intense positive and negative emotions.

In this paper, we present our work on Bro-cam, a system that couples personalization and persuasion to addresses this challenge. Instead of using affective state to adjust gameplay, we describe a game "companion" system that observes the gameplay and the player's postures and then provides humorous feedback. Bro-cam provides its feedback to a player after each match (several matches are part of one gaming session). Players use their body postures and actions to interact with the game. Even when a match of a gaming session is over, the body posture can provide behavioral information about the player. This posture is used by Bro-cam as an indicator of the openness to present adequate feedback. Openness refers to one's personal tendency to take into consideration the possibility of accepting different (or novel) ideas and information, which may lead to a change in one's behavior accordingly. Our system tries to "understand" the player's mood after the game and tries to influence their attitudes by providing encouraging feedback. This is done by taking into account the players' level of openness and profiling them into different personality types. In a prestudy we investigated how rather extrovert or rather introvert players respond to different types of humorous feedback. Subsequently, Bro-cam personalizes the feedback by matching the personality type to their preferences for certain humoristic statements with the aim of increasing the players' motivation for the game.

The two main contributions of this paper are: (1) A system providing empathic game feedback, using the body posture of the players as an indicator of their openness to receive feedback. This approach is unique and novel, because we do not need any other type of user instrumentation, e.g. physiological sensors, to gather information about their level of openness. Furthermore, we can use the same hardware that is also used to interact with the game. (2) A user evaluation demonstrating that adapting game feedback to the players' openness is well received and enriches the overall game experience and the players motivation.

\section{Related work}

Affective computing has been a widely investigated topic for years in humancomputer interaction. The main research challenges and criticisms of affective computing are summarized by Picard [17]. In her cognitive models of emotion [16], the emotions are the central components in the system. In contrast to Picard, Höök and Gaver [11,9] concentrate on using emotions as just one component contributing to the overall design goal of the application or system as also shown by Kuikkaniemi and Nacke $[12,14]$. Many researchers have shown the huge potential of including affective states of users in human-computer interaction within various domains (e.g. games, education, security scenarios) [13]. 
Using physiological data to derive affective states as game input is not entirely new. Recently Nacke [14] demonstrated direct and indirect physiological sensor input to enhance traditional game controls similar to the work of Kuikkaniemi et al. [12]. In addition, Berkovsky [2] developed PLAY,MATE! game design principles which take in account the user's existing engagement to seamlessly motivate them to perform physical activity while playing the game. Earlier works by Dekker [6] and Gilleade [10] provide good overviews of the research area of affective games.

Similar to our work, Gamerini [8] also tailor the game feedback by taking into account the users' personal (energy consumption) behavior. Their work differs from ours by using users' actions to trigger contexualized feedback in order for them to learn about energy conversation. Mapping of personality to the user's behavioral choice has been studied by Tapus et al. [20]. They reported that participants with extroverted personalities had a preference for a robot that challenged them during the training program. Whereas users with introvert personalities preferred the robot that focuses on nurturing praises. In contrast to related work our system passively observes the users' postures (openness) and personalizes the game feedback for motivating them to continue playing the game. We present a new unobtrusive technique to detect the affective states of the player. The same hardware, in our case the Microsoft Kinect, is used to track the gesture input during the game and to give feedback that adapts to the affective state of the users after the game.

Following the abovementioned arguments of Höök and others, we use the affective state of users as input to provide empathic feedback after the gameplay. We see huge potentials in enhancing the game experience with feedback adapted to users' affective state using the same hardware that is already used in motion- or gesture-based controlled games.

\section{Bro-cam System Overview}

The Bro-cam system consists of three main components, which we describe in the following subsections. The first component is the posture classifier that infers the players' openness level. The second component maps the game outcome and player's behavior to appropriate feedback type. This is supported by a pre-study, in which we investigated how rather extrovert or rather introvert players respond to different humoristic statements in winning or losing situations. The feedback statements are then generated in the third component and presented to the player on an additional screen.

\subsection{Inferring openness from postures}

We used a vision-based posture recognition approach to track users' body joints using the Microsoft Kinect depth camera. We implemented a combination of temporal scaling and spatial transformation parameters for 3D body modeling. 


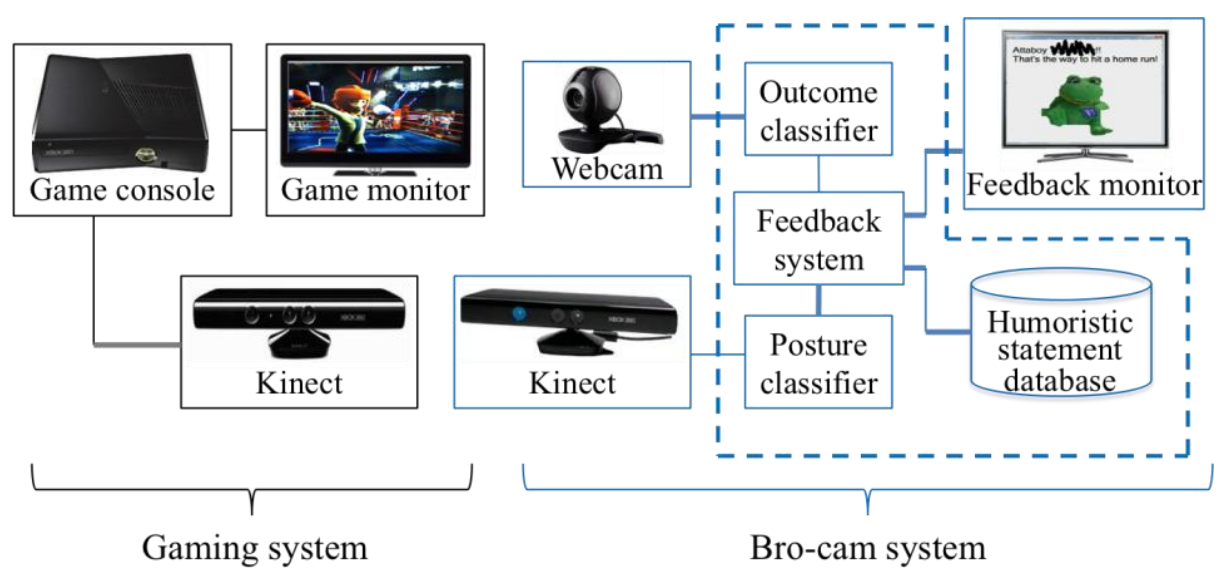

Fig. 1. Bro-cam setup. Two Kinects (stack on top of each other) are facing the player. A webcam is pointing directly at the game monitor to determine for the win-lose outcome. The feedback statements, which are personalized to the player's behavior and win-lose outcome, are then presented to the player on an additional screen.

A 3D body model is tailored for detecting postures based on the OpenNI SDK from PrimeSense. We extracted the angles of fourteen monitored body joints and tracked the movement of these joints every 200 milliseconds. A feed-forward neural network with a Backpropagation Learning algorithm executes the posture recognition algorithms (one for each defined posture).

With this posture classifier approach we are able to successfully classify eleven postures with a mean accuracy of $84.98 \%$ (standard deviation of $7.41 \%$ ) and RMSE of $1.89 \%$. These eleven postures - typically identified in nonverbal communication are (1) Hand-on-chin, (2) Hand-near-hip, (3) Hand-behind-neck, (4) Arm Crossed, (5) Limbs Restrained, (6) Limbs Relaxed, (7) Legs Crossed, (8) Foot Forward (Left), (9) Foot Forward (Right), (10) Lean Forward, and (11) Lean Sideward.

Using a ten-fold cross validation on the training data, the classifier models yield average accuracy rate of $91.03 \%$ for Hand-on-chin, $87.85 \%$ for Hand-near-hip, 85.97\% for Hand-behind-neck, $81.38 \%$ for Arms Crossed, 95.23\% for Limbs Restrained, 83.44\% for Limbs Relaxed, 78.56\% for Legs Crossed, 74.9\% for Foot Forward (Left), $96.06 \%$ for Foot Forward (Right), $86.14 \%$ for Lean Forward, and $74.17 \%$ for Lean Sideward. More details can be found in [19].

To confirm the level of openness conveyed by each of the postures we asked human observers to assess their emotional content. We manually annotated various examples of each of the postures as test patterns and 60 observers (recruited from Amazons Mechanical Turk) rated each test pattern on its level of openness from 1 (not approachable at all) to 7 (very approachable). The faces in the test patterns were pixelated to prevent observers from interpreting emotion from facial expression. The interrater reliability using the Cohen's Kappa statistic, Kappa $=0.59(\mathrm{p}<.001), 95 \%$ CI $(0.468,0.712)$, indicated moderate strength on the agreement among the observers. 


\begin{tabular}{|c|c|c|c|}
\hline $\begin{array}{c}\text { Observers rating } \\
\text { (7-point Likert scale) }\end{array}$ & $\begin{array}{c}\text { Openness } \\
\text { level }\end{array}$ & $\begin{array}{c}\text { Personality } \\
\text { type }\end{array}$ & $\begin{array}{c}\text { Posture } \\
\text { classification }\end{array}$ \\
\hline$M=4.43$, S.D. $=1.54$ & High & Extrovert & $\begin{array}{c}\text { Hand-near-hip, } \\
\text { Limbs Relaxed, } \\
\text { Foot Forward (Right), } \\
\text { Lean Forward }\end{array}$ \\
\hline$M=3.85$, S.D. $=1.57$ & Mid & Ambivert & $\begin{array}{c}\text { Hand-behind-neck, } \\
\text { Limbs Restrained, } \\
\text { Lean Sideward }\end{array}$ \\
\hline$M=3.36$, S.D. $=1.49$ & Low & Introvert & $\begin{array}{c}\text { Hand-on-chin, } \\
\text { Arms Crossed, } \\
\text { Legs Crossed, } \\
\text { Foot Forward (Left) }\end{array}$ \\
\hline
\end{tabular}

Table 1. Profiling of players according to openness level based on human observers rating on 7-point Likert scale. Techniques for character interpretation from Blumenfeld [4] and affect evaluation from Eysenck et al. [7] are used to guide our mapping of openness levels to personality types. Note that we assumed that the interacting entity is located at the direction where the foot forward is point to the right side i.e. Foot Forward (Right).

The between-groups multivariate ANOVA results show that the postures can be grouped into three distinct levels. Postures with high level of openness ( $M=4.43$, SD $=1.54)$ were indeed rated significantly higher than postures with mid level $(M=3.85$, $\mathrm{SD}=1.57), \mathrm{F}(59)=0.476, \mathrm{p}<.001 ; \mathrm{r}=0.002$. Similarly, postures with low level of openness $(\mathrm{M}=3.36, \mathrm{SD}=1.49)$ were rated significantly lower than postures with mid level, $\mathrm{F}(59)=0.467, \mathrm{p}<.001 ; \mathrm{r}=-0.113$. We then related the high level of openness to extroverts with positive affect evaluation [7] who appear approachable, friendly and sociable based on the character interpretation techniques from Blumenfeld [4]. Conversely, a low level of openness is associated with introverts with negative affect evaluation who also appear withdrawn, shy, and reserved, sometimes to the point of inscrutability. We refer to participants who exhibit both extrovert and introvert as ambiverts and associate them with a mid level of openness. Table 1 presents the profiling of players based on the observers rating into different personality type.

\section{2 $\quad$ Feedback mapping}

To investigate how extrovert or introvert players respond to different humoristic statements in different situations we conducted a pre-study. We surveyed pre-test participants to find out their preference through subjective ranking on the appropriate feedback type in winning or losing situations. We asked 65 pre-test participants (recruited from the public fitness center, the cafeteria and the lobby of our university) to fill out the Eysenck Personality Questionnaire [7]. In addition we asked them to rank a compilation of feedback statements for winning or losing situations. 

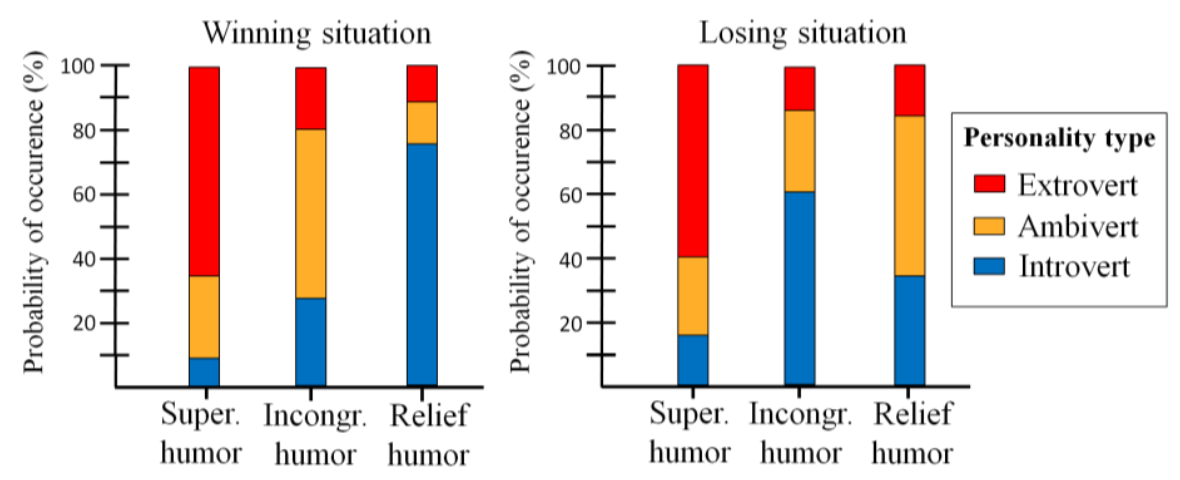

Fig. 2. The preference for each humor during winning and losing situation is presented in term of probability of occurrence as indicated by participants exhibiting one of the three personality types (extrovert, ambivert, introvert).

These humoristic statements are based on three main theories of humor (Superiority, Incongruity, and Relief), which have emerged primarily from psychological studies and research in emotion-oriented systems [18]. The superiority theory suggests that humor is a form of expressing the superiority of one person over another and laughter is triggered by our feelings of superiority with respect to others, e.g. "Attaboy [name]! That's the way to hit a home run!". The incongruity theory suggests that humor is due to the mixing of two disparate interpretation frames for one event e.g. "You must feel as good and refreshed as an egg in the microwave after this beating". The relief theory suggests that humor is a form of bypassing certain censors that prevent us from having uncomfortable thoughts. Thus laughter is induced as a result of release of physical energy, which is built up to deal with disagreeable feelings, e.g. "You buy me a drink and I'll ask your opponent to let you win the next round". We used the findings from the survey to discover the type of humorous feedback that an extrovert (more open) or introvert (less open) would like.

The results on the pre-study, which indicates the preferred humor according to the three personality types, are illustrated in figure 2. We perform the Chi-Square test for independence to determine whether the personality type (extrovert, ambivert, introvert) is actually associated with the preferred humor (Superiority, Incongruity, Relief). In the winning situation, we can conclude from the Chi-Square test results that there is a statistically significant difference between the three personality types and the preferred type of humor. The results for this test are as follows: (1) Superiority humor versus Incongruity humor, $\chi^{2}(4, \mathrm{~N}=65)=73.753, \mathrm{p}=.001, \mathrm{Phi}=.859, \mathrm{~V}=$ .607 , (2) Superiority humor versus Relief humor, $\chi^{2}(4, \mathrm{~N}=65)=17.763, \mathrm{p}=.001$, Phi $=.421, \mathrm{~V}=.298$, and (3) Incongruity humor versus Relief humor, $\chi^{2}(4, \mathrm{~N}=65)=$ $81.476, \mathrm{p}=.001, \mathrm{Phi}=.903, \mathrm{~V}=.638$. The same conclusion to the winning situation can also be drawn for the losing situation. The Chi Square test results for losing situation are as follow: (1) Superiority humor versus Incongruity humor, $\chi^{2}(4, N=65)=$ $44.429, \mathrm{p}=.001, \mathrm{Phi}=.667, \mathrm{~V}=.471$, (2) Superiority humor versus Relief humor, $\chi^{2}(4, \mathrm{~N}=65)=79.184, \mathrm{p}=.001$, Phi $=.890, \mathrm{~V}=.629$, (3) Incongruity humor versus Relief humor, $\chi^{2}(4, \mathrm{~N}=65)=111.947, \mathrm{p}=.001, \mathrm{Phi}=1.086, \mathrm{~V}=.768$. 


\section{Extravert behavior Ambivert behavior Introvert behavior}

\begin{tabular}{|l|l|c|c|}
\cline { 2 - 4 } $\begin{array}{c}\text { Winning } \\
\text { situation }\end{array}$ & Superiority humor & Incongruity humor & Relief humor \\
\cline { 2 - 4 } $\begin{array}{c}\text { Losing } \\
\text { situation }\end{array}$ & Superiority humor & Relief humor & Incongruity humor \\
\cline { 2 - 4 }
\end{tabular}

Table 2. Findings of humor type to the game outcome (winning versus losing) based on exhibited behavior of the player.

Phi and Cramer's V are used as tests of the strength of association. We can see the average Phi $=.804$ and average Cramer's V $=.569$ indicate that the strength of association between the variables is fairly strong. We then create a mapping based on the findings of this pre-study, which is shown in table 2.

\subsection{Feedback presentation}

We developed a feedback system that dynamically changes its output based on the player's extrovert-introvert behavior as indexed by the openness level and whether they won or lost the game. Once the behavior is obtained we need to know the game outcome. We developed an outcome classifier to automatically determine the game outcome by visually processing the game screenshots using a webcam facing the game monitor. This is simply done by analyzing the color histogram of the game monitor as can be seen in figure 1 . The outcome classifier is pre-configured for recognizing the winning and losing color histogram, which in turn, is used to detect the game outcome (i.e. winning or losing status).

Following, we combined the openness level with the game outcome every 3 seconds and added an expiry function to allow extrovert-introvert behavior information to change over a moving window of 30 seconds. The expiry function is intended to reflect the player's dynamic behavioral transition during the progression of the game. With a decreasing marginal utility curve we define the gradual loss in the player's behavioral information. The most appropriate humoristic statement is then selected based on total match strength of the player's behavioral information with the game outcome to create personalized feedback in the form of a sentence. Consequently, the sentence is presented to the player via an avatar (as shown in figure 1). This avatar serves as a social buddy to establish a friendly relationship with the player.

\section{Evaluation}

To evaluate the Bro-cam system for the appropriateness of the feedback we refer to the user satisfaction rather than what the system logs. A user study was conducted with (subjective and objective) quantitative measures. We recruited 36 participants, 
who are used to play video games. The study was successfully completed by 32 participants ( 8 female, 24 male). Data from 4 participants has been discarded, as they did not complete the game due either to personal or health issues. The participants were students or staff at our university and have varying degrees of educational background. Their ages range from 18 to 33 .

\subsection{Apparatus}

The experiment setup is shown in figure 1. The Microsoft Xbox360 gaming console was connected to a 32-inch full HD display. The feedback of the Bro-Cam system was provided on a 42-inch full HD display. Both Kinect sensors were mounted directly below the center of the game monitor. The participants stood about six to eight feet away from the Kinect sensor. The study took approximately 25 minutes for each session. The participants played two matches of Microsoft "Kinect Sports" videogame "Boxing" at the beginner level. The introduction video for the "Kinect Sports" videogame was shown to all participants before they played the game.

\subsection{Procedure}

In the first match of the game, we measured the time to complete the match and established a performance baseline. We then allowed the participants to rest until they felt comfortable to continue with the second match. In the second match of game, we measured again the time taken to complete the match and compared it with the performance baseline to determine the skill level. After each match a feedback from the Bro-cam system was presented to the players. At the end of the session, we conducted a semi-structured interview with the participants to assess qualitatively the impact of empathic feedback. The participants were then debriefed and were allowed to ask any additional questions.

\section{$5 \quad$ Results and analysis}

We perform a set of quantitative analysis on the participants' subjective and objective response, which is obtained from their post interview and game performance respectively. The participants' Likert scale ratings are examined to determine how well the empathic feedback matches the game experience and also how well this feedback motivates them to continue playing with the game.

We assess the strength of agreement from the participants' subjective response using Cronbach's $\alpha$. The result indicates a fairly high strength of agreement score $(\alpha=$ 0.668 ) on the internal consistency for the set of data from the post interview. We then compute for the Levene's Test for Equality of Variances which shows that the variability of the three personality types (extrovert, ambivert, introvert) is the same for the two quantitative measures: (I) feedback is a match to game experience; and (II) feedback is motivating for gameplay. 


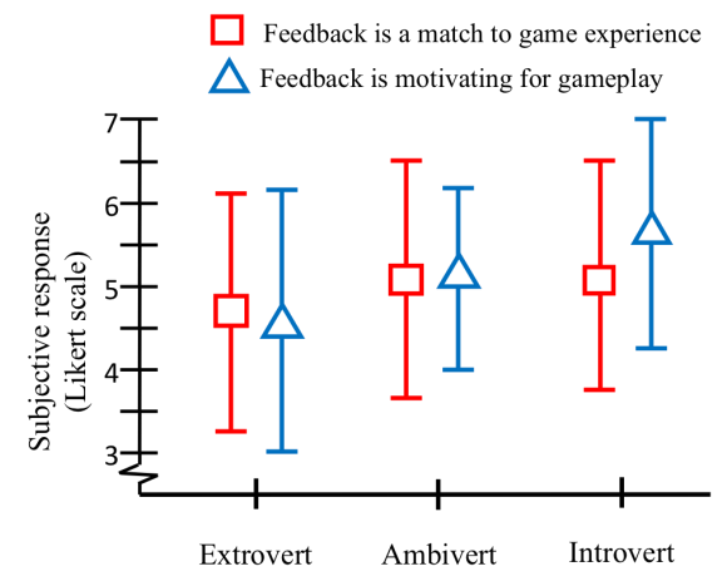

Fig. 3. Likert scale ratings for feedback from players exhibiting different personality type. A Likert scale of 1 to 7 (higher is better) is used. Error bars show standard deviation.

The ANOVA test for $(\mathrm{I})$ shows $\mathrm{F}(29)=.003, \mathrm{p}=.960$, which we can conclude that there is no significant difference in term of how the empathic feedback match their game experience between extrovert $(M=4.731, S D=1.485)$, ambivert $(M=5.091$, $\mathrm{SD}=1.509)$, and introvert $(\mathrm{M}=5.062, \mathrm{SD}=1.436)$ participants. Similarly, the ANOVA test for (II) shows $\mathrm{F}(29)=1.494, \mathrm{p}=.229$, which provide us with the statistical evidence that there is also no significant difference in term of how their motivation for gameplay is affected by the empathic feedback between extrovert $(M=4.654$, $\mathrm{SD}=1.623)$, ambivert $(\mathrm{M}=5.182, \mathrm{SD}=1.097)$, and introvert $(\mathrm{M}=5.687, \mathrm{SD}=$ 1.352) participants.

We used Wilcoxon signed rank test using median equals 4.0 to determine whether there is significant matching of the empathic feedback to the participant's game experience. The extrovert participants with $\mathrm{p}=.0095$ has shown a significant effect $(\mathrm{p}<$ .05 ) on matching the empathic feedback to their game experience. The ambivert participants $(\mathrm{p}=.0015)$ and introvert participants $(\mathrm{p}=.005)$ has shown similar significant effect on matching the feedback to their experience.

Likewise, the results of Wilcoxon signed rank test using median equals 4.0 also show that there is significant improvement on their gaming experience from the empathic feedback. The extrovert participants with $\mathrm{p}=.025$ has shown a significant effect $(p<.05)$ on matching the empathic feedback to their game experience. The ambivert participants $(\mathrm{p}=.001)$ and introvert participants $(\mathrm{p}=.001)$ has shown similar significant effect on matching the feedback to their experience (figure 3 ).

To analyze the objective quantitative measure from the gameplay we focus on the player's skill level, which according to Pfeifer [15], varies the game experience of the players accordingly. After a full analysis on all the players' score and timing, we can categorize 32 participants into two groups which varies according to their skill level: above-average players and below-average players. The skill levels are based on their final scores on each match. Participants with high scores are identified as aboveaverage players and those with lower scores as the below-average players. 


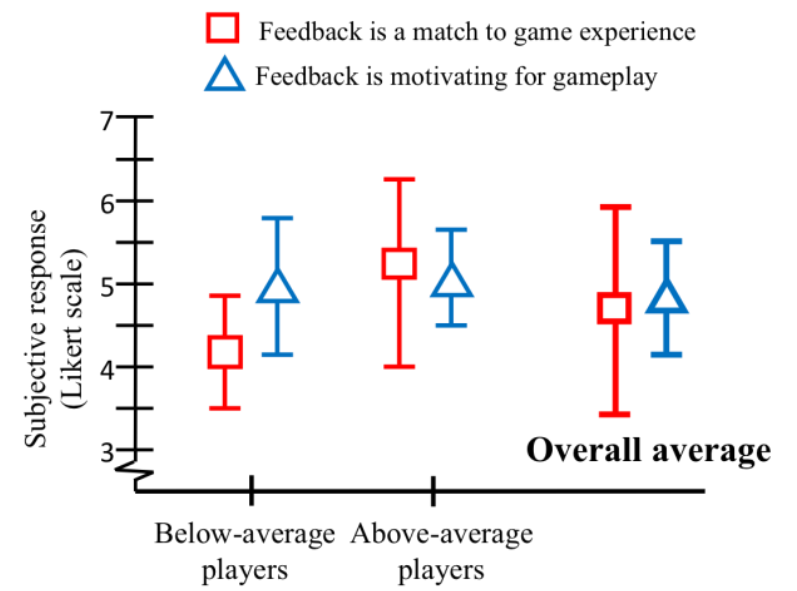

Fig. 4. Likert scale ratings for feedback from players with different skill level. A Likert scale of 1 to 7 (higher is better) is used. Error bars show standard deviation.

The Levene's Test for Equality of Variances, shows that the variability of the two groups is the same. The ANOVA test shows $\mathrm{F}(30)=39.594, \mathrm{p}=.001$. Because of this, we can conclude that there is statistically significant difference between aboveaverage players and below-average players. Using a Mann-Whitney U-test to examine the participants' subjective rating of how the empathic feedback matched their game experience, we found above-average players $(\mathrm{M}=5.286$, $\mathrm{SD}=1.271)$ being significantly different with $\mathrm{p}=.027, \mathrm{U}=60.0, \mathrm{r}=-.703$ to the below-average players $(\mathrm{M}=$ $4.273, \mathrm{SD}=.679$ ). The above-average players thought the feedback is a better match to their gaming experience than below-average players.

We then used Wilcoxon signed rank test using median equals 4.0 to determine whether there is significant matching of the empathic feedback to their game experience. The above-average players with $p=.001$ has shown a significant effect $(p<.05)$ on matching the empathic feedback to their game experience. In contrast, the belowaverage players with $\mathrm{p}=.76$ did not show such significance. However, when we consider all the participants $(\mathrm{M}=4.9375, \mathrm{SD}=1.479)$, they show a significant effect on matching the feedback to their game experience $(\mathrm{p}=.004)$. To determine the effect of feedback on motivating the gameplay, we used a Mann-Whitney U-test to examine how empathic feedback improves their gaming experience. We found that the aboveaverage players are not significantly different $(\mathrm{p}=.669, \mathrm{U}=105.0)$ to the belowaverage players. This indicates that both above-average players and below-average players thought the feedback improves their gaming experience. Using a Wilcoxon signed rank test with median equals 4.0, we show that both above-average players (M $=5.143, \mathrm{SD}=0.526)$ with $\mathrm{p}=.007$ and below-average players $(\mathrm{M}=5.003, \mathrm{SD}=$ 0.848 ) with $\mathrm{p}=.054$ agree that the empathic feedback significantly improves their gaming experience (figure 4). When we consider all participants $(\mathrm{M}=5.093, \mathrm{SD}=$ $0.745)$, the overall results indicated that the empathic feedback $(\mathrm{p}<.05)$ significantly improved their game experience. In summary, the data shows that although all players find the empathic feedback motivating for future matches, below-average players did 
not find that the feedback matched their game experience. In contrast, there exists a high match between the feedback and game experience for above-average players.

\section{Discussion \& Conclusion}

In our study, we observe that the three personality types of players are coherent in term of how the empathic feedback affects their gaming experience. This is validated by the study, which shows that the empathic feedback has a significant effect on its match to game experience and also provide motivation to continue gameplay for all extrovert, ambivert and introvert players.

From the analysis on their game performance, participants who are below-average players experience "a narrow view" during the game. Their objective is on winning the game and their focus is on the game dynamics and coordinating their gross motor skills. Thus the feedback does not seem relevant to their gaming experience. On the other hand, participants who are above-average players enjoy the flow of the game experience [5] (fun experience) and become aware of more details of the game, such as graphics and music. They also find the feedback more relevant. In one instance, Bro-cam showed the following feedback with Incongruity humor type to a participant who had skillfully won the game and exhibited ambivert behavior. "You surely suck as a lover, because you're very good in this game." The participant responded with a surprise grin and exclaimed "That's so true, how did you [Bro-cam] know about it!". Using the body posture of the players as an indicator to their openness and win-lose outcome, we can map different types of humor for participants who exhibited different personality type (i.e. extrovert, ambivert, and introvert) to increase the overall gaming experience. The initial test results show that Bro-cam's empathic feedback is a promising way to improve the gaming experience.

In conclusion, we contribute a new fully-implemented system that analyzes player posture during gameplay and provides empathic feedback to the player based on the posture "openness" and the win-lose status. With that we try to persuade and motivate players to continue with the game. In addition we provide evidence that this type of system can be "well-received" and "enriches the overall game experience." With our approach we also show that it is possible to easily augment the gaming experience for commercial systems by using cheap commodity hardware (webcam and one additional Kinect). Finally, this setup can be used in subsequent research where it is desirable to study users playing commercial titles, rather than using "toy" games that have been customized for a study.

\section{References}

1. Aviezer, H., Trope, Y. and Todorov, A.: Body cues, not facial expressions, discriminate between intense positive and negative emotions. Science 338, 6111, pp. 1225-1229 (2012). 
2. Berkovsky, S., Bhandari, D., Kimani, S., Colineau, N. and Paris, C.: Designing Games to Motivate Physical Activity, In PERSUASIVE 2009 - Persuasive Technology, Fourth International Conference, pp. 37-40. Springer, Heidelberg (2009)

3. Bickmore, T., Gruber, A. and Picard, R. Establishing the computer-patient working alliance in automated health behavior change interventions. Patient Education and Counseling 59, pp. 21-30 (2005)

4. Blumenfeld, R.: Tools and Techniques for Character Interpretation: A Handbook of Psychology for Actors, Writers, and Directors. pp. 115-128, Limelight Editions (2006)

5. Csikszentmihalyi, M.: Flow: The Psychology of Optimal Experience. Harper Perennial, New York (1990)

6. Dekker, A. and Champion, E.: Please Biofeed the Zombies: Enhancing the Gameplay and Display of a Horror Game Using Biofeedback. In Proc of DiGRA (2007)

7. Eysenck, S., Eysenck, H.J. and Barrett, P.: A revised version of the psychoticism scale. Personality and Individual Differences 6, pp. 21-29 (1985)

8. Gamberini, L., Spagnolli, A., Corradi, N., Jacucci, G., Tusa, G., Mikkola, T., Zamboni, L. and Hoggan, E.: Tailoring Feedback to Users' Actions in a Persuasive Game for Household Electricity Conservation. In: M. Bang and E.L. Ragnemalm (eds). Persuasive Technology. Design for health and safety. LNCS, vol. 7284, pp. 100-111, Springer, Heidelberg (2012)

9. Gaver, W.: Designing for emotion (among other things). In Philosophical Transactions of the Royal Society 364, pp. 3597-3604 (2009)

10. Gilleade, K., Dix, A. and Allanson, J.: Affective Videogames and Modes of Affective Gaming: Assist Me, Challenge Me, Emote Me. In Proc of DiGRA, pp. 16-20 (2005)

11. Höök, K.: Affective Loop Experiences - What Are They? In PERSUASIVE 2008 - Persuasive Technology, Third International Conference. pp. 1-12. Springer, Heidelberg (2008)

12. Kuikkaniemi, K., Laitinen, T., Turpeinen, M., Saari, T., Kosunen and I., Ravaja, N.: The influence of implicit and explicit biofeedback in first-person shooter games. In Proc of CHI '10, pp. 859-868, ACM Press (2010)

13. Pantic, M., Caridakis, G., André, E., Kim, J., Karpouzis, K. and Kollias, S.: Multimodal emotion recognition from low-level cues, In: R. Cowie, P. Petta, and C. Pelachaud, (eds), Emotion-Oriented System, pp. 115-132, Springer, Heidelberg (2011)

14. Nacke, L.E., Kalyn, M., Lough, C. and Mandryk, R.L.: Biofeedback game design: using direct and indirect physiological control to enhance game interaction. In Proc of CHI '11. pp. 103-112, ACM Press (2011)

15. Pfeifer, B.: Narrative Combat: Using AI to enhance tension in an Action Game, In: Kirmse, A. (Ed), Game Programming Gems 4, pp. 315-324, Charles River Media (2004)

16. Picard, R.W.: Affective Computing. MIT Press, Cambridge, MA. (2000)

17. Picard, R.W.: Affective Computing: Challenges, International Journal of HumanComputer Studies, vol. 59, no. 1-2, pp. 55-64, Elsevier (2003)

18. Strapparava, C., Stock, O. and Mihalcea, R.: Computational humour. In: R. Cowie, P. Petta, and C. Pelachaud, (eds), Emotion-Oriented Systems, pp. 609-634, Springer, Heidelberg (2011)

19. Tan, S., Schöning, J. Luyten, K. and Coninx, K.: Informing Intelligent User Interfaces by Inferring Affective States from Body Postures in Ubiquitous Computing Environments. In Proc of IUI '13, ACM Press (2013)

20. Tapus, A., Tapus, C. and Matarić, M.J.: User-Robot Personality Matching and Robot Behavior Adaptation for Post-Stroke Rehabilitation Therapy, Intelligent Service Robotics Journal, pp. 169-183 (2008) 